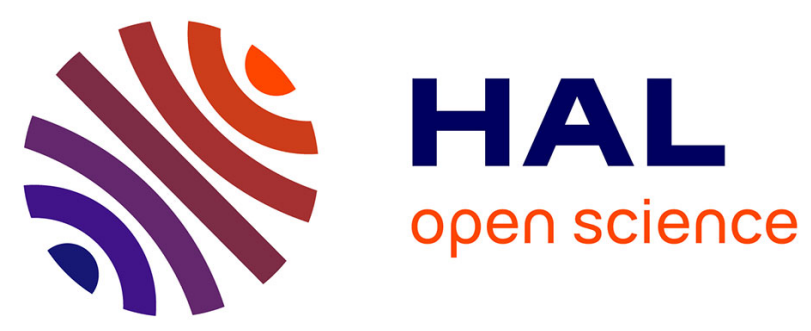

\title{
Compact Waveguide and Guided Beam Pattern Based on the Whispering-Gallery Mode of a Hollow Pillar in a Phononic Crystal Plate
}

\author{
Weitao Yuan, Jinfeng Zhao, Bernard Bonello, Bahram Djafari-Rouhani, \\ Xiaoqing Zhang, Yongdong Pan, Zheng Zhong
}

\section{To cite this version:}

Weitao Yuan, Jinfeng Zhao, Bernard Bonello, Bahram Djafari-Rouhani, Xiaoqing Zhang, et al.. Compact Waveguide and Guided Beam Pattern Based on the Whispering-Gallery Mode of a Hollow Pillar in a Phononic Crystal Plate. Physical Review Applied, 2018, 10 (3), pp.034010. 10.1103/PhysRevApplied.10.034010 . hal-01905839

\section{HAL Id: hal-01905839 \\ https://hal.sorbonne-universite.fr/hal-01905839}

Submitted on 26 Oct 2018

HAL is a multi-disciplinary open access archive for the deposit and dissemination of scientific research documents, whether they are published or not. The documents may come from teaching and research institutions in France or abroad, or from public or private research centers.
L'archive ouverte pluridisciplinaire HAL, est destinée au dépôt et à la diffusion de documents scientifiques de niveau recherche, publiés ou non, émanant des établissements d'enseignement et de recherche français ou étrangers, des laboratoires publics ou privés. 


\title{
Compact waveguide and guided beam pattern based on the whispering gallery mode of hollow pillar in phononic crystals plate
}

\author{
Weitao Yuan ${ }^{1}$, Jinfeng Zhao ${ }^{1 *}$, Bernard Bonello ${ }^{2}$, Bahram Djafari-Rouhani ${ }^{3}$, Xiaoqing Zhang ${ }^{4}$, \\ Yongdong Pan ${ }^{1}$, and Zheng Zhong ${ }^{1}$ \\ ${ }^{1}$ School of Aerospace Engineering and Applied Mechanics, Tongji University \\ 100 Zhangwu Road, 200092, Shanghai, China \\ ${ }^{2}$ Sorbonne Université, UPMC Université Paris 06, INSP UMR CNRS 7588, 4 place Jussieu 75005 \\ Paris, France \\ ${ }^{3}$ Institut d'Electronique, de Microélectronique et de Nanotechnologie, Centre national de la recherche \\ scientifique (CNRS), Unités mixtes de recherche (UMR) 8520, Université de Lille 1, 59650, \\ Villeneuve d'Ascq, France \\ ${ }^{4}$ School of Physics Science and Engineering, Tongji University \\ 1239 Siping Road, 200092, Shanghai, China
}

\begin{abstract}
We report on the experimental evidence of the guiding of the lowest order antisymmetric Lamb mode $\left(\mathrm{A}_{0}\right)$ through a compact waveguide consisting of aligned hollow pillars in between a squarelatticed array of solid pillars on a thin slab. We analyze both numerically and experimentally the guiding achievements and we highlight the role played by the whispering gallery mode of the hollow pillars. In particular, we highlight the evolution of the displacement field pattern behind the waveguide that we relate to the emission of a quadrupolar wave interacting with the surrounding solid pillars. We also show the asymmetric transmission of a compact waveguide based on the same mechanism.
\end{abstract}

\section{INTRODUCTION}

As a result of their capacity to tailor the dispersion of elastic waves, the phononic crystals (PCs) are instrumental in a large number of systems such as acoustic lenses [1], acoustic diodes [2] and even logic gates [3]. Among several other devices, the waveguides are basic but essential components in many integrated acoustic circuits, in particular in the micro-electro-mechanical systems (MEMS) where Lamb and/or surface acoustic waves play a fundamental role. In many studies, they are based on the special modulus of particular equal frequency contours (EFCs) that allow for the wave to propagate in a self-collimating way [4-6]. However, this approach only applies for incident waves with a Gaussian profile and when the waveguide consists of uniform cells [3-5]. Moreover, it may suffer geometrical aberrations if the device is composed of graded structures [6].

Generally, the waveguides are created by inserting into a perfect PC an accurately designed line of defects, giving rise to confined defect modes propagating in the matrix or in the scattering media similarly to a fluid flowing along a channel or in a pipe. Noteworthy achievements have been obtained in this field for different kinds of waves and with different polarizations, including bulk waves [7], Lamb waves[8-10] and surface acoustic waves [11,12]. Actually, the

\footnotetext{
*Corresponding author: jinfeng.zhao@tongji.edu.cn
} 
standard behavior is that the eigenmodes at frequencies inside the band gap of the perfect PC are all the more numerous that the waveguide is broad [8], making these systems very promising for the multiplexing of the elastic waves. Unfortunately, the modes becomes less and less confined in the waveguide as its width increases. Moreover, high energy transmission through a bent waveguide structure remains difficult to achieve even if many efforts have been devoted to solve this problem by, for example, inserting additional scatters at the bends of the guide [11].

Another approach is to use resonators as defects [13-17]. Several designs of resonators have been proposed but since about a decade PC plates constituted by an array of pillars on a homogeneous slab have attracted significant interest across the scientific community. The main interest of these heterostructures lies in the local resonances that may arise at frequencies below the Bragg band gap, giving rise to one or several forbidden bands in a frequency range where the wavelength is much larger than the period of the PC $[9,10,14,15]$. This is a prerequisite for conceiving an elastic metamaterial. Very recently, Jin et al [16] studied waveguides made by substituting solid silicon cylinders for hollow silicon pillars along a line within a perfect array of cylinders on a plate. As an output of this numerical study, they reported on the occurrence of whispering gallery modes (WGM's) in the hollow pillars, as well as their efficient role in the multiplexing properties of the whole structure. They have also shown that hollow pillars feature high order resonances with quadrupolar, hexapolar, and octopolar symmetries and that the quality factors can be enhanced by the introduction of an additional cylinder between the hollow pillar and the plate. However, the influence of these higher order oscillations on the pattern of guided wave through the channel has not been addressed to date [16]. Another important factor that deserves equal attention is the complexity of the fabrication of such stubbed structures on a silicon wafer, by using photolithography processes and isotropic dry etching technologies [18].

In this work, we report on a study both numerical and experimental of a compact waveguide based on hollow pillars erected on a PC plate. We investigate the impact of the quadrupolar WGM's on the transmission of the antisymmetric Lamb wave through the waveguide as well as the distribution of the out-of-plane displacements behind the waveguide, along different directions in the Brillouin zone (BZ). We particularly analyze both numerically and experimentally, the interactions between the quadrupolar WGM's of the hollow pillars and the neighboring solid pillars as well as their consequences on the guided beam pattern. We also show the asymmetric transmission through a compact waveguide based on the same mechanism.

\section{BAND STRUCTURE AND WHISPERING GALLERY MODES}

We first have computed the dispersion curves of a PC plate which unit cell of square symmetry comprises a composite pillar on one of the free surfaces of the plate. To match the actual samples we used in the experiments, we have considered a cylindrical pillar made of steel (labelled A hereafter), glued with epoxy (material B) to a plate made of 6061-T6 aluminum (material C). The outer diameter of the pillar, the thickness of the glue layer, the thickness of the plate, and the lattice constant were respectively (in mm): $d_{1}=5, h_{\mathrm{B}}=0.135$ [19], $t_{\mathrm{C}}=1.5$, and $a=6$. Both the height of the steel pillar $h_{\mathrm{A}}$ and the inner diameter $d_{2}$ of the entire pillar (steel and epoxy) were left as free parameters. As regards the physical parameters of the different materials we used for the Young's modulus (in GPa) $E_{\mathrm{A}}=226, E_{\mathrm{B}}=5.1$, and $E_{\mathrm{C}}=$ 
67.7; for the Poisson's ratios $v_{\mathrm{A}}=0.29, v_{\mathrm{B}}=0.38$, and $v_{\mathrm{C}}=0.35$; and for the mass densities (in g.cm $\left.{ }^{-3}\right) \rho_{\mathrm{A}}=7.75, \rho_{\mathrm{B}}=1.2$, and $\rho_{\mathrm{C}}=2.7[19-21]$.

The dispersion curves have been computed using the finite element code COMSOL Multiphysics ${ }^{\circledR}$. The result for a PC with solid pillars (i.e. $\left.d_{2}=0\right)$ and $h_{\mathrm{A}}=2.4 \mathrm{~mm}$ is shown as red open circles in Fig. 1(a). With this set of parameters, two narrow stop bands open up in the intervals $97.0-103.7 \mathrm{kHz}$ and $109.8-114.2 \mathrm{kHz}$ as well as a much broader band gap at higher frequency, in between 166.5 and $245.7 \mathrm{kHz}$. As already pointed out in Ref. [22], this result is quite different from what is obtained in the absence of the epoxy layer, i.e. when the pillars $\mathrm{A}$ are directly fastened on the plate $\mathrm{C}\left(h_{\mathrm{B}}=0\right.$, not shown here). Actually, when including the epoxy layer in the computations the first six branches displayed in Fig. 1(a) are significantly downshifted. This is the consequence of the low Young's modulus of epoxy which is lower than that of steel and aluminum by one order of magnitude. A similar behavior has already been demonstrated for SAWs propagating in a set of steel pillars regularly glued on a semi-infinite aluminum substrate [19].

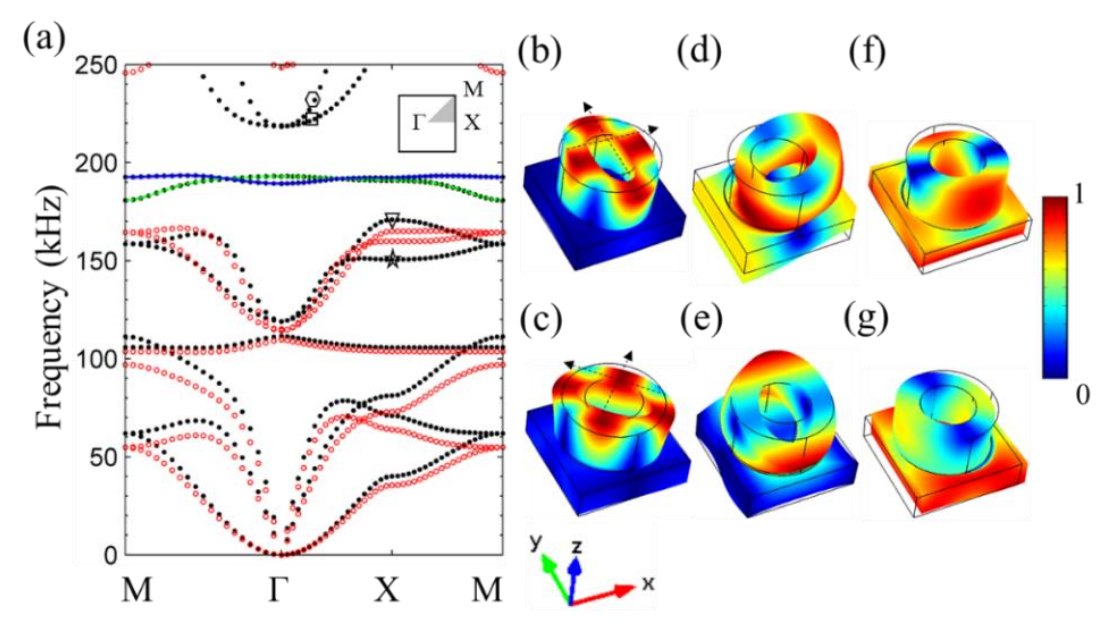

FIG. 1. (a) Band structure of PC plates with either solid (red circle lines, $d_{2}=0$ ) or hollow pillars (black dotted lines, $d_{2}=2.8 \mathrm{~mm}$ ). The inset shows the reduced BZ of the square lattice. Normalized displacement and deformation at point X of the BZ for (b) WGM1 and (c) WGM2 along the blue and green solid lines in panel (a), respectively. Black arrows indicate the polar axes of WGM's. Normalized distribution at the points highlighted by the (d) star, (e) triangular, (f) square, and ( $g$ ) hexagon symbols. The dimensions are $a=6 \mathrm{~mm}, h_{A}=2.4 \mathrm{~mm}, h_{B}=0.135 \mathrm{~mm}, t_{C}=1.5 \mathrm{~mm}$, and $d_{1}=5 \mathrm{~mm}$.

We then computed the band structure for the unit cell comprising hollow pillars A and B on the slab with the inner diameter $d_{2}=2.8 \mathrm{~mm}$ and the height of the steel pillar kept unchanged, $h_{A}=$ $2.4 \mathrm{~mm}$. The result is shown as black dotted lines in Fig. 1(a). As a general trend the branches at low frequency are upshifted within the whole BZ, as compared to the system with solid pillars. This tendency is not found any more for the fifth branch and above. However the most noteworthy feature is the two new flat branches, highlighted by the blue and green lines in Fig. 1(a), appearing around $190 \mathrm{kHz}$ within the stop band of the PC plate with solid pillars. Fig. 1(b) and Fig. 1(c) show the displacement field distribution normalized to unity $u_{t}$, and the deformation of the unit cell for both these modes at the X point of the BZ, in the blue and the 
green lines respectively. This allows to clearly identify both modes as quadrupolar WGM's [23]. The mode in Fig. 1(b), hereafter labelled as WGM1, has two polar axes (black arrows) along the $x$ and $y$ axes respectively, while the mode WGM2 in Fig. 1(c), features two polar axes rotated by $45^{\circ}$ relatively to the $x$ and $y$ axes. The two branches are almost flat and cover the common range 190.9-192.3 kHz along $\Gamma X$, crossing each other at $f=191.8 \mathrm{kHz}$. The displacement fields $u_{t}$ displayed in Figs. 1(b) and 1(c) clearly reveal the strong localization on the upper end of the pillar of both WGM1 and WGM2. The displacements are maximum on top of the pillar and exponentially decrease down to 0.16 (Fig. 1(b)) or 0.18 (Fig. 1(c)) on the foot. No displacement affects the plate. In addition, because of their quadrupolar symmetry, the wavelength for both WGM's corresponds to half the acoustic path along the perimeter $\pi<d>$, where $<d>=\left(d_{1}+d_{2}\right)$ $/ 2$ is the mean diameter [16]. Although all the materials from which is made the PC are isotropic, whatever is the direction in the BZ the two resonant modes do not occur at the same frequency except at the two points where the dispersion curves cross each other (Fig. 1(a)). This is the consequence of the good coupling through the slab and the epoxy layer of the vibrations in neighboring resonators. It is interesting to note that when the resonators and the substrate are made of anisotropic materials, e.g. silicon, the wavelengths of both WGM's are still equal to each other since they derive from geometrical considerations. However, the two corresponding branches in the BZ are generally well separated [16], reflecting the anisotropy of the sound velocities.

To complete this analysis, Figs. 1(d) and 1(e) show the displacement field at the lower edge of the Bragg band gap, at point X of the BZ (points labelled by a star and a triangular symbol in Fig. 1(a) respectively). These modal shapes which both are corresponding to bending modes polarized along the $x$ axis and the $y$ axis respectively, affect the pillar as well as the slab, albeit in a different way. Actually, the deformation of the plate being antisymmetric at the lower frequency of the two (Fig. 1(d)) suggests an easy coupling with the lowest order antisymmetric Lamb $\left(\mathrm{A}_{0}\right)$ mode that shares the same symmetry. In contrast the deformation shown in Fig. 1(e) exhibits a complex shape involving displacements along the $y$ - and $z$-axes of almost equal amplitude and a much smaller component along the $x$-axis. Lastly, Figs. 1(f) and 1 (g) depict the displacement field of the unit cell at the upper edge of the high frequency band gap, at the points of the BZ marked by square and hexagon symbols in Fig. 1(a). Both modes correspond to the bending of the hollow pillar either along the $y$ axis (Fig. 1(f)) or along the $x$ axis (Fig. 1(g)), whereas the slab features in both cases a shear horizontal deformation, as evidenced by the large amplitude of both in-plane components $u_{x}$ and $u_{y}$, as compared to the very small out-of-plane component $u_{z}$. It can be anticipated from this analysis that none of these modes can couple with $\mathrm{A}_{0}$ mode.

In order to optimize the achievements of the waveguides, we paid special attention to the influence of the height of the steel hollow pillar $h_{\mathrm{A}}$ on the eigenfrequencies of the resonators. The variations of the eigenfrequencies against $h_{\mathrm{A}}$, computed at point $\mathrm{X}$ of the $\mathrm{BZ}$, are displayed as dotted lines in Fig. 2(a). In the computations, the inner diameter $d_{2}$ remained unchanged $\left(d_{2}\right.$ $=2.8 \mathrm{~mm}$ ). The eigenfrequencies of both modes WGM1 (blue solid line) and WGM2 (red solid line) decrease as $h_{\mathrm{A}}$ increases, with a rate of variation that diminishes as $h_{\mathrm{A}}$ increases. This trend is correlated to the decay of the displacement field $u_{t}$ downward the pillar's top surface for both WGM's. Actually, the larger $h_{\mathrm{A}}$ the smaller $u_{t}$ at the foot of the pillar, as shown by the color 
scale for the lines overlapping the square symbols in Fig. 2(a). In other words increasing $h_{\mathrm{A}}$ allows to better confine the elastic energy in the pillar and to reduce the coupling between the WGM's and the waves propagating in the slab. Correlatively, the eigenfrequencies of both WGM's are all the less affected by the wave propagating in the slab when $h_{\mathrm{A}}$ becomes larger, and the difference between the two eigenfrequencies reduces. From this point of view, we can also infer that the width of WGM's branch decreases when the height $h_{\mathrm{A}}$ increases.

Examples of a band structure when $h_{\mathrm{A}}$ has a value as large as $5 \mathrm{~mm}$ are shown in Fig. 2(b) either for solid pillars (red circle lines, $d_{2}=0$ ) or for hollow ones (black dotted lines, $d_{2}=2.8 \mathrm{~mm}$ ). As previously, the band structure corresponding to the PC plate with hollow pillars features two flat branches, highlighted by the blue and green lines in Fig. 2(b), within the broad band gap that opens up in the interval $130-218 \mathrm{kHz}$ when the pillars are solid. The flatness of both branches, as compared to their counterparts in Fig. 1(a), gets enhanced due to the reduced coupling between WGM's and the wave in the slab as noted above. This reflects a group velocity that is close to zero at any frequency in this branch and in turn, the strong localization in the pillars of the elastic energy carried by the two WGM's. Meanwhile, the two branches still cross each other along $\Gamma \mathrm{X}$ when $h_{\mathrm{A}}=5 \mathrm{~mm}$, just as they do at $f=191.8 \mathrm{kHz}$ when $h_{\mathrm{A}}=2.4 \mathrm{~mm}$, but at the slightly smaller frequency $f=181.5 \mathrm{kHz}$. In this case, the normalized displacement field $u_{t}$ computed either at the lower edge of the band gap (triangular and star symbols in Fig. 2(a)) or at the upper edge (square and hexagon symbols) is similar to its respective counterpart noted by the same symbols in Fig. 1(a) (not shown here).

(a)

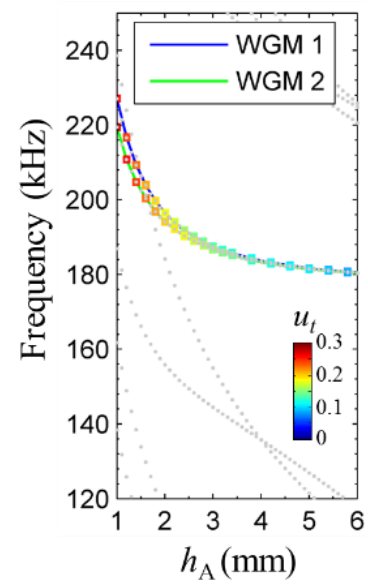

(b)

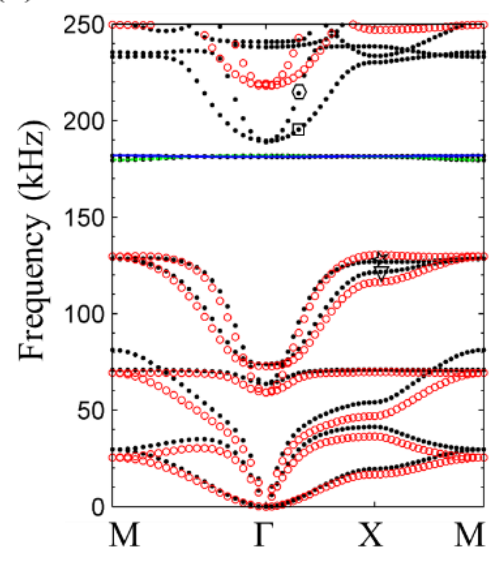

FIG. 2. (a) Eigenfrequencies at point $X$ of the BZ (gray dotted lines) against $h_{A}$ for the $P C$ with hollow pillars $\left(d_{2}=2.8 \mathrm{~mm}\right)$. Inset: color scale of the amplitude $u_{t}$ normalized to the amplitude computed on the thin slab next to the foot of the pillar. (b) Band structure of the solid (red circle lines, $d_{2}=0$ ) or hollow (black dotted lines, $d_{2}=2.8 \mathrm{~mm}$ ) PC plate when $h_{A}=5 \mathrm{~mm}$. In both panels, $W G M 1$ and $W G M 2$ are indicated by the solid blue and green lines, respectively. The geometrical parameters are (in mm) $a=6, h_{B}=0.135, t_{C}=1.5$, and $d_{1}=5$.

\section{EXPERIMENTAL SETUP, SAMPLES DETAILS, AND BAND STRUCTURE OF SUPPERCELL}


Our experimental setup combines the air-coupled method [24] and the laser ultrasonic technique [19]. To generate the lowest-order antisymmetric Lamb mode $\mathrm{A}_{0}$ in the slab, upstream the PC, we utilized an unfocused air-coupled transducer (ACT) as an emitter. It had a diameter of $32 \mathrm{~mm}$; the central frequency was $f_{0} \approx 177 \mathrm{kHz}$ and the band width $\sim 32 \%$. This ACT was attached to a rotary stage so that it could be turned in the $x-z$ plane. According to Snell's law, to generate $\mathrm{A}_{0}$ mode one needs tuning the incident angle $\theta$ to satisfy the relationship $\theta=\sin ^{-}$ ${ }^{1}\left(s_{x} \cdot c\right)$, where $c$ stands for the sound velocity in the air and $s_{x}$ denotes the slowness of $\mathrm{A}_{0}$ mode in the plate [24]. The optimal incident angle was computed to be $\theta=13.8^{\circ}$. The ACT was attached to a high power generator (JPR-600C, electrical voltage $300 \mathrm{~V}$ ) that provided ultrasonic RF pulses of five cycles to the aluminum plate, over an area comparable to the surface of the ACT. In return, $\mathrm{A}_{0}$ mode was excited on the plate that expanded along the transverse direction, to cover a fan-shaped region originating from the impinged area with an apex angle of about $60^{\circ}$. We set the ultrasonic source along the $x$ axis at $\sim 14 \mathrm{~cm}$ away from the pillars, far enough from the PC for the wave could be considered as a plane wave with a Gaussian profile in the central area of the fan-shaped region where the line source was located.

Concerning the detection, we used a laser Doppler vibrometer (LDV, Polytec vibrometer OFV 2570) to record the out-of-plane displacement $u_{z}$ as a function of time and space. Each measurement point resulted from the averaging over 512 scans digitized by an oscilloscope (DPO 4102B) at the sampling frequency of $1.25 \mathrm{GHz}$. This measurement channel allowed for a signal to noise $(\mathrm{S} / \mathrm{N})$ ratio of $\sim 6.4$, going up to $\sim 15$ after filtering out the white noise. We used this setup to measure the transmission coefficient $T=U / U_{0}$ through the PC, where $U_{0}$ stands for the amplitude of the source signals recorded on the pure aluminum substrate at a distance off the emitter the same as the pillars, whereas $U$ corresponds to the amplitude of the signals recorded at the outlet of the waveguides. Actually, the incident wave featuring a broad frequency range, further filtering of both $U_{0}$ and $U$ was necessary to derive $T$ from our measurements. To do this, we used a Gaussian profiled window with a very narrow bandwidth centered at the frequency of interest [25].

In order to achieve wave guiding based on WGM's, we have investigated two designs of compact waveguides differing from one another by their orientation in the BZ. The first waveguide was featuring two lines of pillars in a square lattice on a thin slab, with $\Gamma \mathrm{X}$ being parallel to the $x$ axis (see Fig. 3(b)). The second device was composed of three lines of pillars, also in a square lattice but with ГМ parallel to the $x$ axis (see Fig. 4(b)). These structures will be designed hereafter as the $\Gamma \mathrm{X}$ sample for the former and the $\Gamma \mathrm{M}$ sample for the latter. In both configurations, the waveguides are conceived in such a way that a line of defects comprising two identical hollow pillars were inserted in between the solid pillars array, along the $x$ axis. The period was $a=6 \mathrm{~mm}$, the plate thickness $t_{\mathrm{C}}=1.5 \mathrm{~mm}$, the glue thickness $h_{\mathrm{B}}=0.135 \mathrm{~mm}$, and the outer diameter $d_{1}=5 \mathrm{~mm}$, as before. The hollow pillars had an inner diameter $d_{2}=2.8$ $\mathrm{mm}$. The choice for the height of the hollow pillars $h_{\mathrm{A}}=2.4 \mathrm{~mm}$ was a compromise between a well separation of the WGM's from any other normal mode of the hollow PC plate on one hand, and keeping a relatively strong coupling between the hollow pillars and the thin slab (i.e. a relatively large $u_{t}$ at the foot of the hollow pillars, see Fig. 2(a)) on the other hand. The solid pillars the geometry of which was chosen to allow for the best possible confinement of the $\mathrm{A}_{0}$ mode within the guide, had a height $h_{\mathrm{A}}=5 \mathrm{~mm}$ and not of $2.4 \mathrm{~mm}$ because of the broad gap 
obtained with this dimension. This point was further confirmed in a preliminary experiment where the transmission $T$ of $\mathrm{A}_{0}$ mode through the $2 a$ wide array of solid pillars along $\Gamma \mathrm{X}$ was measured. We measured $T<-30 \mathrm{~dB}$ in between $140 \mathrm{kHz}$ and $240 \mathrm{kHz}$ with $h_{\mathrm{A}}=5 \mathrm{~mm}$, to be compared with $T>-25 \mathrm{~dB}$ in between $140 \mathrm{kHz}$ and $230 \mathrm{kHz}$ with $h_{\mathrm{A}}=2.4 \mathrm{~mm}$. This strong capacity of $5 \mathrm{~mm}$ high solid pillars to filter $\mathrm{A}_{0}$ mode is comparable to known results obtained with $2 a$ wide PC strip where a similar mechanism is involved [22], i.e. the overlapping of the local resonance of a single solid pillar to the Bragg scattering mechanism.

Both samples were built on a homogeneous aluminum slab using some epoxy adhesive to glue the solid and the hollow pillars that were elaborated through wire-electrode cutting. This electro-erosion process allows for both high accuracy and reproducibility of the nominal dimensions. Besides the parameters $a, h_{\mathrm{B}}$, and $t_{\mathrm{C}}$ were all in very good agreement with their nominal values upon sample completion. Each of the two samples spreads over $120 \mathrm{~mm}(20 a)$ along the $y$ axis, and they were erected on a plate large enough to have no edge effects. All the experiments were performed at least 24 hours after sample completion and therefore no aging effect of the epoxy resin were observed.

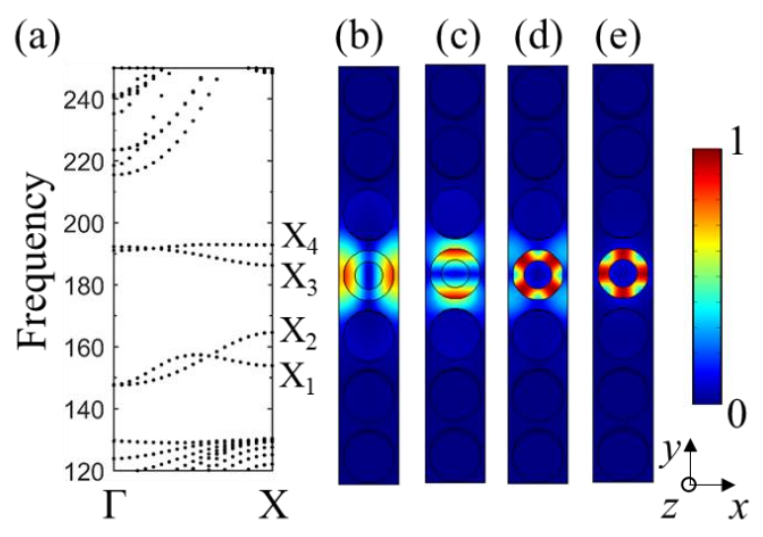

FIG. 3. (a) Band structure calculated with the $a \times 7 a$ supercell shown in (b) where a hollow pillar $\left(h_{A}=2.4 \mathrm{~mm}, d_{2}=2.8 \mathrm{~mm}\right)$ stands on the substrate in between six solid pillars $\left(h_{A}=5 \mathrm{~mm}\right)$. (b) - (e) Normalized distribution of $u_{t}$ at points $X_{1}, X_{2}, X_{3}$, and $X_{4}$ of the BZ indicated in panel (a).

Before we present the experimental results, let us return to the band structure computed using the supercell method [8-11]. Figure 3(a) shows the band structure of the $\Gamma \mathrm{X}$ sample calculated by using the $a \times 7 a$ supercell repeated along both the $x$ and $y$ axes, whereas Fig. 3(b) depicts the supercell where one hollow pillar $\left(h_{\mathrm{A}}=2.4 \mathrm{~mm}, d_{2}=2.8 \mathrm{~mm}\right)$ is flanked on both sides by three solid pillars $\left(h_{\mathrm{A}}=5 \mathrm{~mm}\right)$. The other geometrical parameters are the same as before. Obviously, Fig. 3(a) exhibits four branches in the band gap of the PC plate with solid pillars: the first two branches fall within 148 and $165 \mathrm{kHz}$ whereas the other two are found between 186 and $193 \mathrm{kHz}$. Figures 3(b) - 3(e) show the normalized distribution of $u_{t}$ for each of these four branches at point $\mathrm{X}$ of the BZ, i.e. at the points labeled $\mathrm{X}_{1}-\mathrm{X}_{4}$ in Fig. 3(a). As can be seen from Figs. 3(b) and 3(c), the polarizations are oriented along $x$ or $y$ axes and couple well to the substrate. This highlights the confinement of the propagative modes in the linear defect. On the other hand, Figs. 3(d) and 3(e) show the WGM2 and WGM1 in the hollow pillar, so that both WGM's keep well confined in the waveguides. Indeed, these modes confined in the line defect 
are all the signatures of the modes in the PC plate made only of hollow pillar as can be seen by a direct comparison of their frequencies/modal shapes with those presented in Fig. 1.

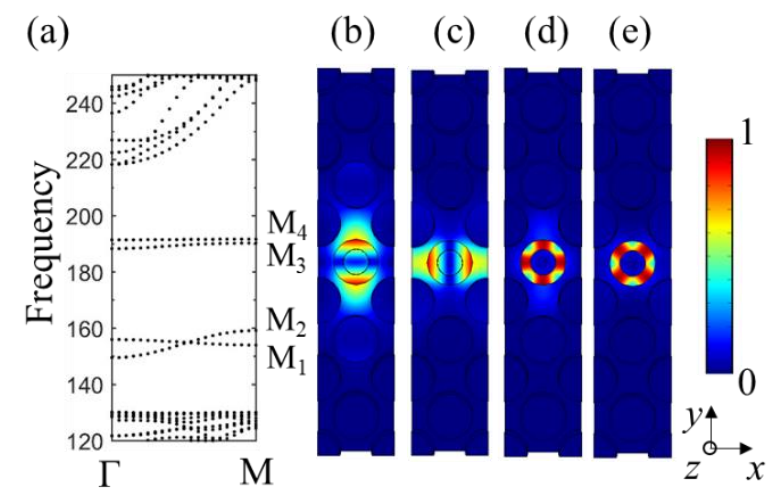

FIG. 4. (a) Dispersion curves computed using the $\sqrt{2} a \times 5 \sqrt{2} a$ supercell (b) featuring a hollow pillar $\left(h_{A}=2.4 \mathrm{~mm}, d_{2}=2.8 \mathrm{~mm}\right)$ glued in the central position on the slab while the other solid pillars $\left(h_{A}=5 \mathrm{~mm}\right)$ are arranged symmetrically along the transverse direction. (b) - (e) Normalized distribution of $u_{t}$ at point $M_{1}-M_{4}$ of the BZ in order in panel (a).

Regarding the ГМ sample, we computed the band structure (Fig. 4(a)) by using the $\sqrt{2} a \times$ $5 \sqrt{2} a$ supercell shown in Fig. 4(b). This supercell repeats itself along both the $x$ and $y$ axes with $\Gamma \mathrm{M}$ parallel to the $x$ axis and one hollow pillar $\left(h_{\mathrm{A}}=2.4 \mathrm{~mm}, d_{2}=2.8 \mathrm{~mm}\right)$ in between solid pillars $\left(h_{\mathrm{A}}=5 \mathrm{~mm}\right)$. The other geometrical parameters are unchanged. As can be seen from Fig. 4(a), four branches appear in the stop band of the PC plate with solid pillars. The normalized distributions of $u_{t}$ at point $\mathrm{M}$ of the BZ, i.e. at the points labelled by $\mathrm{M}_{1}-\mathrm{M}_{4}$ in Fig. 4(a), are shown in Figs. 4(b)-4(e) respectively. In Figs. 4(b) and 4(c), the propagative mode is emphasized by the hollow pillar polarized along the $y$ or $x$ axis, respectively, whereas in Figs. 4(d) and 4(e), the hollow pillar displays the displacement field corresponding to WGM1 and WGM2. As a result, both the propagative modes and WGM's remain confined in the linear defect, as what happens to their counterpart in the $a \times 7 a$ supercell, in spite of the branch modulation of the corresponding modes caused by the symmetry of the lattice of solid pillars.

\section{EXPERIMENTAL RESULTS AND ANALYSIS}

\section{A. Preliminary results and analysis}




\section{(a)}



(b)

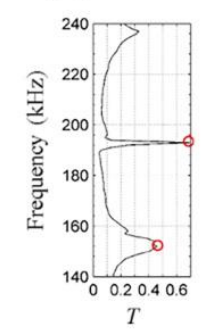

(c)

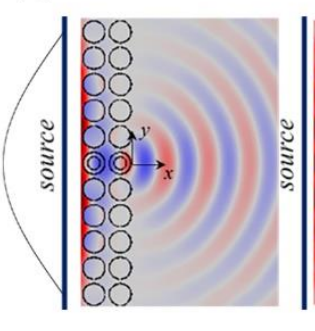

(d)

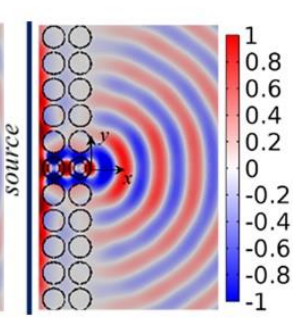

FIG. 5. (a) Transmission spectra measured along a line at $x=6 \mathrm{~mm}$ behind $\Gamma X$ sample. The circle and diamond lines correspond to the profiles at the peak in the first and second transmitted spots. (b) Transmitted amplitude computed at $y=0$. Normalized distribution of $u_{z}$ at the frequencies noted by a circle in panel (b) at (c) $f=154 \mathrm{kHz}$ and (d) $f=193 \mathrm{kHz}$. The component $u_{z}$ is normalized to the amplitude of the incident wave. In panel (c), the thin solid line stands for the profile of the applied force polarized along the $z$ axis on the line source (bold line) in front of pillars.

We show in Fig. 5(a) the transmission spectrum as a function of the position along the $y$ axis, recorded at a distance $x=6 \mathrm{~mm}$ behind the $\Gamma \mathrm{X}$ sample. The first thing to notice is the almost total extinction of the signal for $|y|>10 \mathrm{~mm}$ whatever is the frequency in the band gap of the PC made of solid pillars, i.e. [140-220 kHz]. This illustrates the efficiency of this structure to serve as a filter for $\mathrm{A}_{0}$ Lamb mode, as shown also above by using the $2 a$ wide array of solid pillars with $h_{\mathrm{A}}=5 \mathrm{~mm}$. The second remarkable fact comes from the two spots emerging on the central axis at $y=0$ and at frequencies $f=169 \mathrm{kHz}$ and $f=191 \mathrm{kHz}$. The transmitted amplitude in the first spot extends within an effective distance $(T>0.1)|y|<12 \mathrm{~mm}$ and gradually decreases from the center; it covers the frequency domain $150-178 \mathrm{kHz}$, the maximum $T_{\max }=$ 0.50 occurring when $f=169 \mathrm{kHz}$. This pass band, exactly matches the domain delimited by the two branches associated to the bending modes in the band structure of the PC made of hollow pillars (Fig. 1(a)). The associated displacement fields are shown in Figs. 1(d) and 1(e). Another spot in the transmission spectrum appears for $|y|<8 \mathrm{~mm}$, in the range 184-204 kHz. The frequency $f=191 \mathrm{kHz}$ at which the transmissivity is maximum, $T_{\max }=0.70$, is that of the WGM branches in Fig. 1(a).

We then have simulated with a finite element code the wave propagation through the waveguide, using the same procedure as for the experiments: the transmission coefficient $T$ is derived from the maximum amplitude computed along the $y$ axis, $6 \mathrm{~mm}$ behind the outlet interface and then normalized to the amplitude of the incident wave. To mimic $\mathrm{A}_{0}$ mode source, we set a transverse 
line (11 $a$ long) at a distance of $7.5 \mathrm{~mm}$ before the inlet interface, upon which we applied a force polarized in the $z$ axis with a Gaussian profile along $y$ axis (see the thin solid line in front of the crystal in Fig. 5(c)). According to our former experimental results [19], the standard deviation was fixed to $40 \mathrm{~mm}^{-1}$. Perfect matched layers (PMLs) were bound to the substrate to eliminate boundary reflections. [16] We then made the calculation over the interval 140-240 kHz. As can be seen in Fig. 5(b), one broad transmission peak occurs at $f=154 \mathrm{kHz}\left(T_{\max }=0.46\right)$, in fairly good agreement with the frequency measured experimentally $(f=169 \mathrm{kHz})$. A much sharper peak occurs at $193 \mathrm{kHz}\left(T_{\max }=0.69\right)$, in very good agreement with the experimental result $(f=$ $191 \mathrm{kHz}$ ). It is noteworthy that $T$ keeps almost the same profile as the one displayed in Fig. 5(b) whatever is the position of the source within an interval of $5 \lambda(f=180 \mathrm{kHz})$ in front of the waveguide, regardless of the fluctuations of $T_{\max }$ in between 0.63 and 0.7 around $f=193 \mathrm{kHz}$.

Figures 5(c) and 5(d) show the distribution of $u_{z}$ at the frequencies where the transmission is maximum. In both cases, the guiding by the pair of hollow pillars is clearly evidenced by the displacement fields they undergo, as shown in Figs. 5(c) and 5(d). Actually, the bending mode polarized along the $x$ axis allows for the transmission at $154 \mathrm{kHz}$ whereas the deformation of the hollow pillars is that of WGM1 at $193 \mathrm{kHz}$. In both cases, the transmission gets possible owing to the strong coupling with the substrate through the epoxy layer. Furthermore, symmetry conditions are necessary for the normal modes to be excited by the incident antisymmetric Lamb wave. This is why the bending mode polarized along $y$ axis (Fig. 1(e)) cannot couple with the incident wave and does not affect the transmission through the wave guide. Similarly, at $f=193 \mathrm{kHz}$ WGM2 whose polar directions are at an angle of $45^{\circ}$ to the $x$ - and $y$ axes (Fig. 1(c)), is not observable in simulations [16]. Neither of them is taken into account below. 
(a)

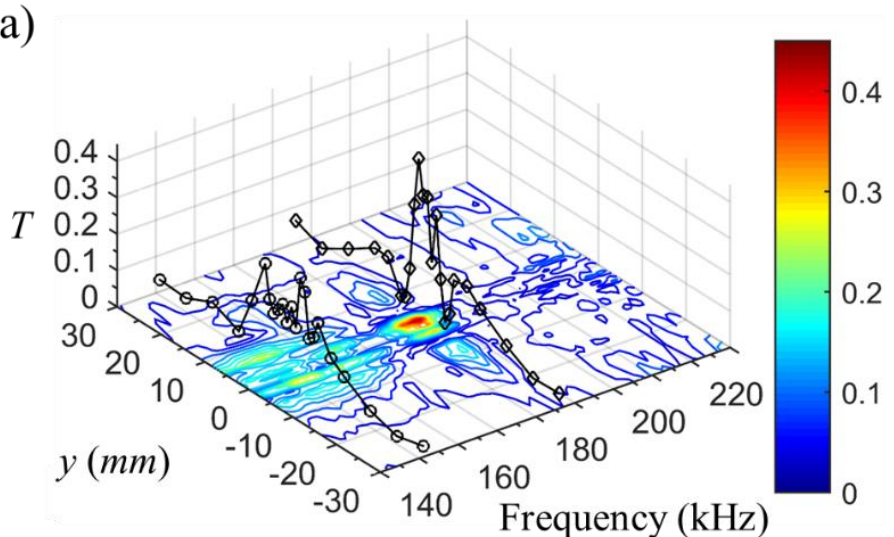

(b)

(c)

(d)

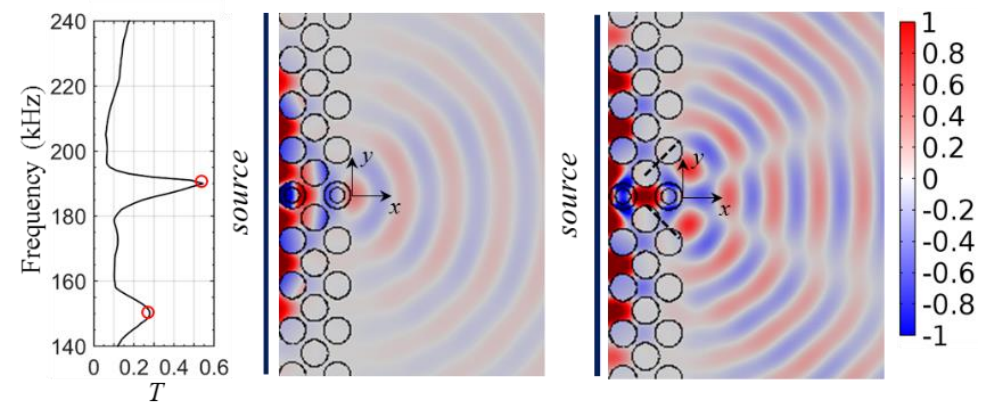

FIG. 6. (a) Transmission spectra measured along a line at $x=6 \mathrm{~mm}$ behind the $\Gamma M$ sample. The circle and diamond lines correspond to the profiles at the peaks in the first and second transmitted spots. (b) Transmitted amplitude computed at $y=0$. Normalized distribution of $u_{z}$ at the frequencies noted by a circles in panel (b) at (c) $f=152 \mathrm{kHz}$ and (d) $f=190 \mathrm{kHz}$. The component $u_{z}$ is normalized to the amplitude of the incident wave.

We used the same approach to investigate the transmission through the $Г \mathrm{M}$ sample. Figure 6(a) displays the transmission spectrum along the $y$ axis measured at a distance of $6 \mathrm{~mm}$ behind the PC. One can easily distinguish in this figure the transmission $(T>0.1)$ of vibrations at frequencies in the interval $140-170 \mathrm{kHz}$, in a region spreading over $|y|<11 \mathrm{~mm}$. The mapping of $u_{z}$ in this spot, although not being exactly symmetrical with respect to the $x$ axis decreases from the central area, where the maximum $T_{\max }=0.28$ is reached at $f=151 \mathrm{kHz}$, to the lateral sides. This is consistent with the computed spectrum plotted in Fig. 6(b) where $T$ features a broad peak centered at $153 \mathrm{kHz}\left(T_{\max }=0.28\right)$, in good agreement with the measured profile. The small discrepancies between the experimental results in Fig. 6(a) and the numerical results in Fig. 6(b) may arise from slight imperfections of the samples, such as the disorder in the position of the pillars, the eccentricity of the air hole... For the same reasons, such small discrepancies between the experimental results in Fig. 5(a) and the numerical results in Fig. 5(b) also exist for the $\Gamma \mathrm{X}$ sample. To further illustrate the transmission of the elastic waves at this frequency, we show in Fig. 6(c) the simulated normal component $u_{z}$. As previously, the two hollow pillars in the guide feature displacements associated to the bending vibration polarized along the $x$ axis. Here again, due to symmetry considerations the bending mode polarized along $y$ direction is not excited into vibration and therefore it does not play any role in the transmission of the Lamb wave. 
A second peak $\left(T_{\max }=0.55\right)$ emerges at $191 \mathrm{kHz}$ in the transmitted spectrum computed on the central axis of the sample at $x=0$ and displayed in Fig. 6(b). Its position within the spectrum well agrees with the one measured experimentally at $f=186 \mathrm{kHz}$ and displayed in Fig. 6(a). This peak can be unambiguously imputed to WGM1 that falls at $190 \mathrm{kHz}$ for this geometry. This is further corroborated by the displacement field on top of the resonators shown in Fig. 6(d). Another interesting characteristic of the amplitude pattern is the two valleys at about $\pm 23^{\circ}$ to the $x$ axis separating the main peak in between $y=-3 \mathrm{~mm}$ and $y=4 \mathrm{~mm}\left(T_{\max }=0.44\right)$ from two secondary maxima, resulting from interferences between the waves scattered by the solid pillars surrounding the waveguide. These secondary maxima, also visible on the diamond line drawn in Fig. 6(a), extend over $-15 \mathrm{~mm}<y<-6 \mathrm{~mm}\left(T_{\max }=0.18\right)$ and $9 \mathrm{~mm}<y<14 \mathrm{~mm}$ $\left(T_{\max }=0.12\right)$ respectively. Overall, the peaks cover the range $180-192 \mathrm{kHz}$ in the frequency domain that is to say the range covered by the WGM's in Fig. 1(a).

To summarize, the guiding of Lamb waves based on their coupling with WGM's and a transmission $T_{\max } \approx 0.7$ have been achieved both numerically and experimentally. These prototype structures may have applications in many fields. For example, putting one hollow pillar at the corner of L- or T- shaped defect $[10,11]$, or erecting several designed hollow pillars as a combination, would allow to create more configurable acoustic circuits and therefore to achieve more complicated functions based on the WGM's, including multiplexing [16], wave bending [27], liquid sensors [28], and one-wave transmission [29] (see also below). Using semiconductors as the constitutive material of well dimensioned structures would allow creating interaction between phonons and photons [30], suggesting in turn potential applications in the control of optical waves, e.g. the emission of photon by tuning the acoustic wave through the WGM's. This is of significant importance when considering that WGM's have been widely used in optics [31]. To increase the transmissivity, which is an important issue for applications, it is essential to enhance the coupling between the WGM's and the wave in the substrate, including but not limited to, the use of single-phased material.

\section{B. Two mechanisms: pattern of the guided waves and transmitted amplitude}

Analyzing the field scattered by a single pillar, either solid or hollow, can help in understanding the relationship between the pattern observed behind the PC and the guiding mechanism. To this end, we have considered a numerical model in which an antisymmetric Lamb wave with a Gaussian profile impinges a $2.4 \mathrm{~mm}$ high steel pillar bonded to a $1.5 \mathrm{~mm}$ thick aluminum slab with the epoxy adhesive. The geometries of the pillar and the gluing layer were the same as before; the slab was surrounded by PMLs to prevent from any reflection on the edges of the slab [26]. 

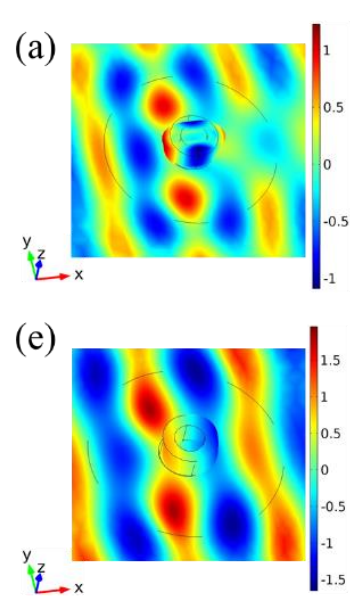
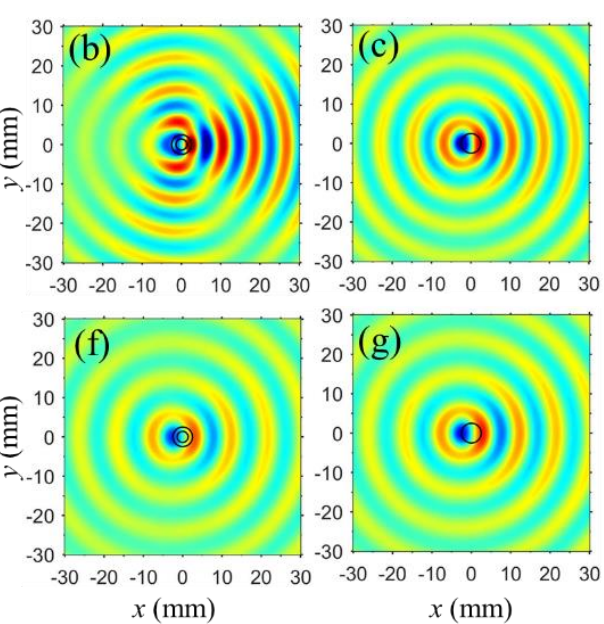

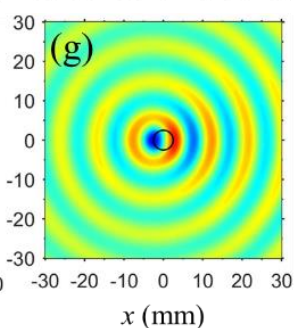

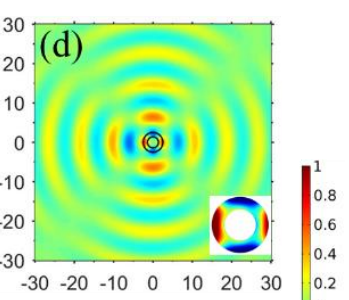

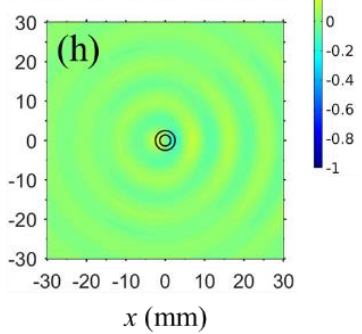

Fig. 7. (a) Normalized distribution of $u_{z}$ and deformation of a hollow pillar upon the incidence of $A_{0}$ mode at $f=192 \mathrm{kHz}$. Normalized scattered field in the slab by (b) the hollow or (c) the solid pillar at $f=192 \mathrm{kHz}$. The black lines are for the edges of the pillars. (d) Scattered field obtained by subtracting $u_{z}$ in panel (c) from that in panel (b); the inset is a zoom of $u_{z}$ on the top surface of the hollow pillar. The dimensions of both the solid and hollow pillars are $h_{A}=2.4 \mathrm{~mm}$ and $d_{1}=$ $5 \mathrm{~mm}$; the inner diameter of the hollow pillar is $d_{2}=2.8 \mathrm{~mm}$. $A_{0}$ is incident along the positive direction of $x$ axis. The displacement shown in panels (a) to (d) are all normalized to the amplitude of the incident wave. Panels (e) to (h) are the counterparts at $f=150 \mathrm{kHz}$ of panels (a) to (d).

We show in Fig. 7(a) the total displacement field $u_{z}$ computed at $f=192 \mathrm{kHz}$, normalized to the amplitude of the incident wave, highlighting the excitation of WGM1 in the hollow pillar. As long as we are dealing with the linear regime, the total field can be viewed as the sum of the incident wave and the wave reemitted by the pillar at resonance [26]. Therefore, the latter term can be determined by subtracting the former one from the total field. The result, further normalized to the amplitude of the incident wave, is displayed in Fig. 7(b). It is interesting to notice that the displacement caused in the plate by the pillar at resonance does not have the fourfold symmetry characteristic of WGM1, neither at the pillar's foot nor in the slab far away from. Instead, the normal displacement $u_{\mathrm{z}}$ features a complex mapping that we have analyzed as follows. First, one should notice that the diameter of the hollow pillar is close to the operating wavelength $\left(d_{1} \approx 0.60 \lambda\right)$ and therefore the scattering processes get dominated by the ballistic regime. Then we have computed the displacement field scattered by a solid pillar with $h_{\mathrm{A}}=2.4$ $\mathrm{mm}$, glued on the slab. As shown in Fig. 7(c), the scattered field as well as the displacement on top of the pillar have the dipolar symmetry resulting from the bending vibrations of the pillar.

Lastly, we subtracted the scattered field caused by the solid pillar (Fig. 7(c)) from the one caused by the hollow pillar (Fig. 7(b)); we obtained the pattern shown in Fig. 7(d) which clearly features the quadrupolar symmetry attached to WGM1. This is further supported by the deformation on top of the pillar displayed in the inset in Fig. 7(d) that displays the same symmetry. This inset and Fig. 7(d) also show that the phases on top and on foot of the pillar are identical. It is also interesting to notice that the wavelength of the wave in the plate is that of $\mathrm{A}_{0}$ mode at $f=192 \mathrm{kHz}$, i.e. $\lambda=8.3 \mathrm{~mm}$ and that the amplitude along the main axes close to the pillar is comparable to that of the incident wave. To summarize, this analysis shows that at the 
frequency of WGM1, a single hollow pillar acts as a secondary elastic source that reemits along $x$ and $y$ axes in the plate, an antisymmetric Lamb wave that superimposes to the waves scattered by the hard obstacle that the pillar is. Unsurprisingly, a Lamb wave impinging a periodic lines of hollow pillars undergoes Bragg scattering on each elements which leads to destructive interferences inhibiting the transmission of the incident wave, whatever is the direction in the BZ. In contrast, the WGM's being coherently excited by the incident wave, guarantee the transmission.

The transmission peak at $f=150 \mathrm{kHz}$ results from another mechanism. Indeed, we show for comparison in Fig. 7(e) the total displacement field $u_{z}$ around a hollow pillar as $\mathrm{A}_{0}$ mode at this frequency propagates in the plate. No strong localization of energy is observed in the pillar. The fields scattered either by a hollow or by a solid pillar $2.4 \mathrm{~mm}$ high are displayed in Figs. 7(f) and $7(\mathrm{~g})$ respectively. In both cases, the scattered beam propagates forward and keeps confined within a relatively narrow angular sector. For either of these scatters the dispersion in the PC plate along $\Gamma \mathrm{X}$ and $\Gamma \mathrm{M}$, displayed in Fig. 1(a), features at $f=150 \mathrm{kHz}$ two propagative branches allowing for the guiding of $\mathrm{A}_{0}$ mode through the channel. Therefore, the hollow pillar cannot have a big influence as confirmed by the minor differences between the scattering displacements caused by the hollow or the solid pillar shown in Fig. 7(h).

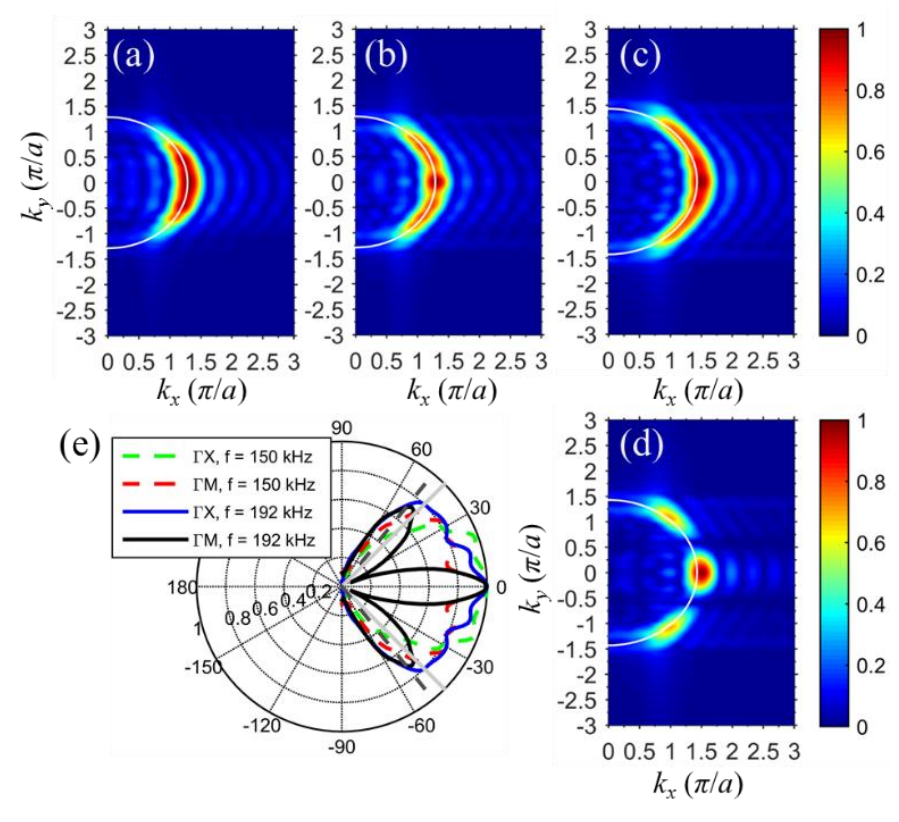

FIG. 8. Normalized $2 D$ Fourier transform of the computed $u_{z}$ in the rear of waveguide, at $f=150$ $\mathrm{kHz}$ for (a) $\Gamma X$ and (b) $\Gamma M$ sample, and at $f=192 \mathrm{kHz}$ for (c) $\Gamma X$ and (d) $\Gamma M$ sample. The white lines indicate the equal frequency contour (EFC) of $A_{0}$ mode in the homogeneous slab. (e): Normalized Fourier component taken along the EFC at $f=150 \mathrm{kHz}$ for $\Gamma X$ (green dashed line) and $\Gamma M$ (red dashed line) sample, and at $f=192 \mathrm{kHz}$ for $\Gamma X$ (blue solid line) or $\Gamma M$ (black solid line). All figures are normalized separately.

To further investigate the directivity of the beam pattern and its relationship with the wave scattered by a single pillar, we have calculated the $2 \mathrm{D}$ Fourier transform of the simulated displacement field downstream the waveguide at $f=150 \mathrm{kHz}$ and $192 \mathrm{kHz}$ for both the $\Gamma \mathrm{X}$ 
sample (Figs. 8(a) and 8(c) respectively) and the ГM sample (Figs. 8(b) and 8(d) respectively). The corresponding equi-frequency contour (EFC) for $\mathrm{A}_{0}$ mode in a slab free from any inclusion is also drawn as a white solid line in Figs. 8(a)-(d). It is noteworthy to observe that whatever the numerical situation, the components of the Fourier transform well spread along the EFC, showing that no mode conversion occurs upon transmission through the guide. Actually, because of the folding of the dispersion curves that ensues the periodicity in the waveguide, the wavenumber of either the scattered wave or WGM1 always satisfies $|k|<\pi / a$. On the other hand, although not fully accurate here since the wavelength is not much larger than the period $(\lambda=$ $8.3 \mathrm{~mm}$ at $f=192 \mathrm{kHz}$ ), the Snell's law may help estimating the spreading of the elastic beam downstream the waveguide. This law states the continuity of $k_{y}$ at the interface between the PC and the substrate. The $y$ component of the wave vector verifies $\left|k_{y}\right|<\pi / a$ out of the waveguide and therefore the elastic beam at $f=150 \mathrm{kHz}$ (resp. $f=192 \mathrm{kHz}$ ) should keep confined within an angular sector spreading over $\pm 51^{\circ}$ (resp. $45^{\circ}$ ) on both sides of the $x$ axis.

One can get a clearer picture on the mapping of the displacement field in the substrate by computing the amplitude of the Fourier component against the angle for both frequencies. The results at $150 \mathrm{kHz}$ are shown in Fig. $8(\mathrm{e})$ as a green dashed line and red dashed line for the ГX and $Г \mathrm{M}$ samples respectively; the blue solid line and the black solid line are for the amplitude at $192 \mathrm{kHz}$ in the $\Gamma X$ and $\Gamma \mathrm{M}$ sample respectively. This figure shows that components $\left|k_{y}\right|=$ $0.5 \pi / a$ result from the scattering at the right edge of the PC, leading to an angular sector of $\sim \pm 50^{\circ}$ (resp. $\sim \pm 61^{\circ}$ ) at $150 \mathrm{kHz}$ for $Г X$ (resp. ГM) sample. The larger angular sector for the $\Gamma M$ sample can easily be explained by the position of the surrounding solid pillars that let a wider opening angle in comparison to the $\Gamma X$ sample. This well explains the fan-shaped pattern downstream the waveguide, except for the transmission through the $Г M$ sample at $192 \mathrm{kHz}$ which is much more directional and points towards three well defined directions.
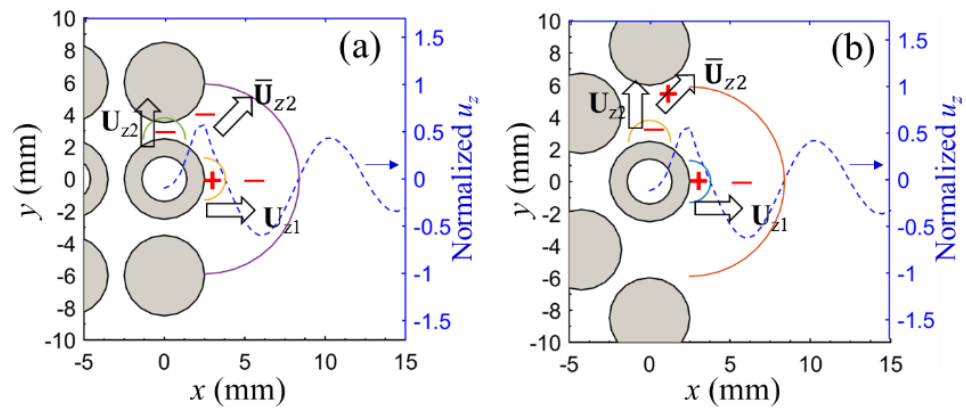

FIG. 9. Scheme of the scattered wave induced by WGMI assumed to be the sum of the wave along the $x$ axis $\boldsymbol{U}_{z 1}$ and the $y$ axis $\boldsymbol{U}_{z 2}$, and scheme of its interaction with the solid pillars nearby in (a) $\Gamma X$ sample and (b) $\Gamma M$ sample. $\boldsymbol{U}_{z 1}$ and $\boldsymbol{U}_{z 2}$ starts with opposite phases at the foot of the hollow pillar. In both panels, the dashed line indicates the amplitude of normalized $u_{z}$ (right scale).

To explain this result, let us separate the scattered field caused by WGM1 and displayed in Fig. $7(\mathrm{~d})$, into the wave scattered along the $x$ axis, namely $\mathbf{U}_{\mathrm{zl}}$, and the one scattered along $y$ axis $\mathbf{U}_{z 2}$, as shown in Figs. 9(a) and 9(b). Then, we define the initial time as the moment when the amplitude is maximum at the foot of the hollow pillar on the right of the guide. This is the situation described by the dashed lines in Figs. 9(a) and 9(b) which can be formally written as $\mathbf{U}_{z 1}=\mathbf{U}^{0}{ }_{\mathrm{z} 1} \cos \left(k x-\pi d_{1} / \lambda\right)$. The wavelength being $\lambda=8.3 \mathrm{~mm}$ at $f=192 \mathrm{kHz}, \mathbf{U}_{\mathrm{z} 1}$ takes negative 
values at a distance of $\sim 4.6 \mathrm{~mm}$ from the origin. Correlatively, the displacement field displayed in the inset in Fig. 7(d) shows that $\mathbf{U}_{\mathrm{z} 2}$ measured at the foot of the hollow pillar is minimum, and hence negative, when $\mathbf{U}_{\mathrm{z} 1}$ is maximum. For both samples, the source $\mathbf{U}_{\mathrm{z} 1}$ reemits to the right a wave within an angular sector of about $45^{\circ}$ on both sides of $x$ axis (see Fig. 7(d)) where it encounters no obstacle. As for the wave $\mathbf{U}_{\mathrm{z} 2}$, regardless the sample, it encounters at short distance a solid pillar that can be seen as an infinitely rigid cylindrical scatter. As a result, the wave $\mathbf{U}_{\mathrm{z} 2}$ gets scattered to a direction satisfying $\left|k_{y}\right|=\pi / a$ and forming an angle of $\sim 51^{\circ}$ to the $x$ axis. We denote this scattered wave as $\overline{\mathbf{U}}_{z 2}$. When the free space between the hollow pillar and the neighboring solid pillars along the $y$ axis is less than $\lambda / 4(\lambda=8.3 \mathrm{~mm}$ when $f=192 \mathrm{kHz})$, $\overline{\mathbf{U}}_{z 2}$ and $\mathbf{U}_{z 1}$ have the same sign and constructively interfere. This is the situation in the $\Gamma \mathrm{X}$ sample (Fig. 9(a)) where the free space is equal to $1 \mathrm{~mm}$ i.e. $0.12 \lambda$ allowing for the displacement field displayed in Fig. 5(d) as well as for the radiation pattern in Fig. 8(e) (blue line) or the EFC in Fig. 8(c). Conversely, when the free space falls in between $\lambda / 4$ and $3 \lambda / 4, \overline{\mathbf{U}}_{z 2}$ and $\mathbf{U}_{z 2}$ have opposite signs. This is what happens in the $\Gamma \mathrm{M}$ sample, where the free space is equal to $3.5 \mathrm{~mm}$, i.e. $0.42 \lambda$, as described in Fig. 9 (b). $\overline{\mathbf{U}}_{z 2}$ and $\mathbf{U}_{z 1}$ having opposite signs they destructively interfere giving rise to a transmission dip at an angle of about half of $51^{\circ}$, i.e. $25.5^{\circ}$, in good agreement with the calculated one $23^{\circ}$.

\section{Experimental results through long waveguides}
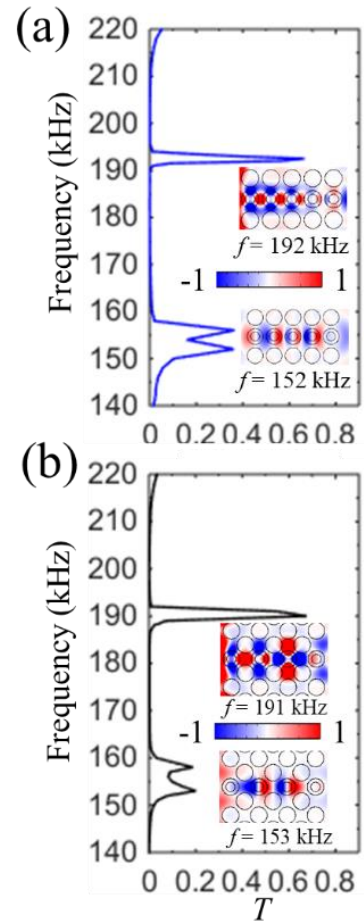

(c)

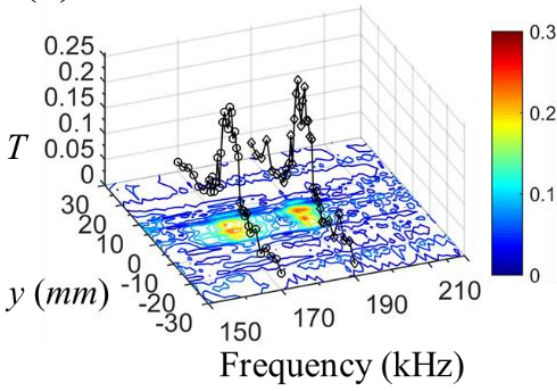

(d)

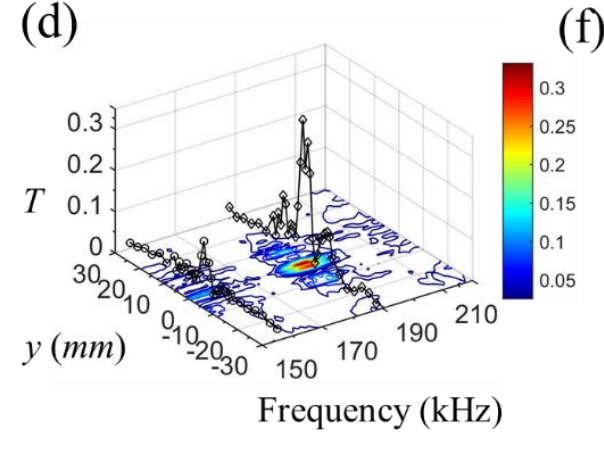

(e)

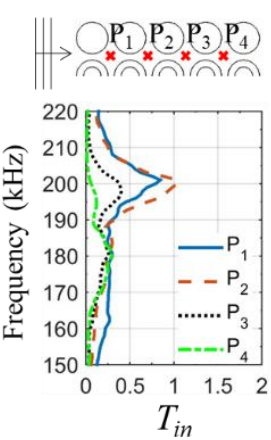

(f)



FIG. 10. Calculated transmissivity of $A_{0}$ mode through (a) $\Gamma X$ sample with 5 pillars, or (b) $\Gamma M$ sample featuring 7 lines of pillars. Insets: distribution of $u_{z}$ normalized to the amplitude of the incident wave computed on the top surface of the slab, within both the first and second passing bands. Measured transmissivity of $A_{0}$ mode along the transverse line $6 \mathrm{~mm}$ behind the (c) $\Gamma X$ or (d) $Г M$ samples, and the normalized signal amplitude $T_{i n}$ recorded at the points $P_{1}-P_{4}$ (cross symbols in the upper inset panel) within the (e) ГХ or (f) ГM sample. 
After the demonstration of wave guiding through the short $Г \mathrm{X}$ and $\Gamma \mathrm{M}$ samples, let us turn to long partners, in order to give clearer evidence thanks to insights of the signals recorded inside the samples. To this end, simulations of the transmission through a guide in the $Г \mathrm{X}$ sample consisting of five lined up hollow pillars are shown in Fig. 10(a) [15,16]. The sharp peak at 192 $\mathrm{kHz}$ resulting from the resonant WGM1 remains unaffected by the lengthening of the guide, in contrast with the passband centered on $152 \mathrm{kHz}$ which is a little narrower and less intense than it is in the short waveguide. Identical observations can be made for the peak at $191 \mathrm{kHz}$ and the passband centered on $153 \mathrm{kHz}$ when computing the transmission spectrum with a $Г \mathrm{M}$ sample featuring a guide with seven hollow pillars (Fig. 5(b)).

Actually, the transport of elastic energy at the frequency of WGM1 is a recursive process that includes the excitation of the local resonance and the forward reemission of $\mathrm{A}_{0}$ mode: when the first hollow pillar is set into vibration by the incident wave, it reemits $\mathrm{A}_{0}$ mode along the $x$ axis which excites in turn the second hollow pillar into vibration on its resonant mode. This process repeats up to the last pillar in the guide. Besides this process, the antisymmetric Lamb mode is also reemitted along the $y$ axis upon the generation of WGM1. Whether in the $\Gamma \mathrm{X}$ or the $Г \mathrm{M}$ sample, this wave is back reflected by the surrounding solid pillars to the hollow pillar that is excited again on WGM1. Most part of the elastic energy is guided that way to the outlet interface. However, as the frequency of WGM1 is very sensitive to the diameter $d_{2}$ of the inner hole [16,17], the variations of $T$ caused by the fluctuations of this parameter because of manufacturing issues are less important in the short waveguide.

As shown by the inset in Fig. 10(a), the mode at $f=152 \mathrm{kHz}$ also propagates forward up to the last hollow pillar in the waveguide but in that case no reemitted waves are involved in the transmission process. Rather, at this frequency the device is a conventional guide in which the waves keep confined through scattering and reflections on the surrounding solid pillars.

TABLE I. Experimental features of the first transmitted spot.

$$
T_{\max } \quad f \text { at } T_{\max } \quad \text { Frequency }
$$

$(\mathrm{kHz}) \quad$ interval $(\mathrm{kHz})$

\begin{tabular}{llll}
\hline$\Gamma \mathrm{X}(2$ lines $)$ & 0.5 & 169 & $150-178$ \\
\hline$\Gamma \mathrm{X}(5$ lines $)$ & 0.22 & 170 & $163-180$ \\
\hline$\Gamma \mathrm{M}(3$ lines $)$ & 0.28 & 153 & $150-170$ \\
\hline$\Gamma \mathrm{M}(7$ lines $)$ & 0.12 & 155 & $150-160$ \\
\hline
\end{tabular}

TABLE II. Experimental features of the second transmitted spot.

\begin{tabular}{llll}
\hline & $T_{\max }$ & $\begin{array}{l}f \text { at } T_{\max } \\
(\mathrm{kHz})\end{array}$ & $\begin{array}{l}\text { Frequency } \\
\text { interval }(\mathrm{kHz})\end{array}$ \\
\hline$\Gamma \mathrm{X}(2$ lines $)$ & 0.7 & 191 & $184-204$ \\
\hline$\Gamma \mathrm{(5}$ lines $)$ & 0.26 & 190 & $186-197$ \\
\hline$\Gamma \mathrm{M}(3$ lines $)$ & 0.55 & 191 & $180-192$ \\
\hline$\Gamma \mathrm{M}(7$ lines $)$ & 0.37 & 187 & $181-196$ \\
\hline
\end{tabular}


We also have fabricated $\Gamma \mathrm{X}$ and $\Gamma \mathrm{M}$ samples featuring 5 and 7 lines of pillars respectively. Figures 10(c) and 10(d) show the transmission spectrum mapped along the transverse line 6 $\mathrm{mm}$ behind the $\Gamma \mathrm{X}$ or $\Gamma \mathrm{M}$ sample. In Fig. 10(c), two remarkable transmitted spots spread on the central axis whereas $T$ decreases gradually to the lateral sides. The first spot that corresponds to $T_{\max }=0.22$ at $f=170 \mathrm{kHz}$ falls within the passband $163-180 \mathrm{kHz}$. This is consistent with its counterpart measured in the short $\Gamma X$ sample and shown in Fig. 5(a). It is likely that $T_{\max }$ decreases and the passing range reduces because of the reflections and sample imperfections arising with the increase in the length of the waveguide. In Fig. 10(c), the second spot for which $T_{\max }=0.26$ appears at $f=190 \mathrm{kHz}$ and covers the interval $186-197 \mathrm{kHz}$, as it was the case in the short $\Gamma \mathrm{X}$ sample, except that $T_{\max }$ is smaller and that the transmitted range gets narrower. The second spot shall be definitely attributed to the WGM's that appear in the same range. Tables I and II summarize the frequency range, $T_{\max }$ and the relevant frequencies derived from experiments, for the first and second transmitted spots respectively.

Behind the long ГМ sample, the first transmitted spot with $T_{\max }=0.12$ is measured over the interval $150-160 \mathrm{kHz}$ centered at $f=155 \mathrm{kHz}$, as shown in Fig. 10(d). With the increasing length of the waveguide, this spot gets narrower and less intense in comparison to its counterpart in Fig. 6(a). In Fig. 10(d), the second transmitted spot with $T_{\max }=0.37$ at $f=187$ $\mathrm{kHz}$ within the passband $181-196 \mathrm{kHz}$, is similar to its counterpart behind the short $\Gamma \mathrm{M}$ sample in Fig. 6(a). Further, $T_{\max }$ decreases because of the sample imperfections as the waveguide length increases. However, the frequency width of $15 \mathrm{kHz}$ is close to the value of $12 \mathrm{kHz}$ measured for the short ГM sample. Nevertheless in Fig. 10(d), the second transmitted spot keeps the trefoil shape pattern with two valleys at about $\pm 34^{\circ}$ to the $x$ axis separating the main peak whose $-3 \mathrm{~mm}<y<2 \mathrm{~mm}\left(T_{\max }=0.37\right)$ from two secondary maxima. These secondary maxima extend over $-11 \mathrm{~mm}<y<-8 \mathrm{~mm}\left(T_{\max }=0.12\right)$ and $7 \mathrm{~mm}<y<9 \mathrm{~mm}\left(T_{\max }=0.12\right)$ respectively.

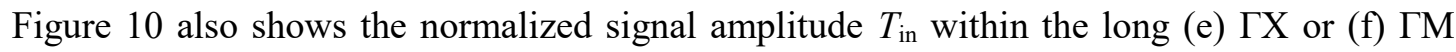
sample at the points $\mathrm{P}_{1}-\mathrm{P}_{4}$ (cross symbols in upper inset panel). The coefficient $T_{\text {in }}$ is derived in the same way as the coefficient $T$. The points $\mathrm{P}_{1}-\mathrm{P}_{4}$ are located at the central positions in between pillars on the horizontal line above the hollow pillar defect. As a result of the lengthening of the waveguide, the general trend for $T_{\text {in }}$ depicted in Fig. 10(e) is decreasing as the points are closer to the outlet. In particular around $f=200 \mathrm{kHz}, T_{\text {in }}$ features a peak at $\mathrm{P}_{1}$ or $\mathrm{P}_{2}$ but a small value at $\mathrm{P}_{4}$, in good agreement with the distribution of $u_{z}$ calculated when $f=192$ $\mathrm{kHz}$ and shown in the inset of Fig. 10(a). Therefore, this must be related to the WGM's. However, around $f=180 \mathrm{kHz}, T_{\text {in }}$ keeps an almost constant value (see Fig. 10(e)), close to the one calculated when $f=152 \mathrm{kHz}$ and displayed in the inset of Fig. 10(a), within the spot whose width gradually decreases from $\mathrm{P}_{1}$ to $\mathrm{P}_{4}$. The propagative mode accounts for this transmission. It should be noticed that both the propagative mode and the WGM's exist at these points. This can lead to possible energy overlap, so that the frequency of WGM's or propagative mode presents a shift in comparison to their counterparts in Fig. 10(c).

In Fig. 10(f), although $T_{\text {in }}$ has a large value at the entrance point $\mathrm{P}_{1}$, it features similar profiles at $\mathrm{P}_{2}-\mathrm{P}_{4}$ with a relatively large amplitude within a narrow spot centered at $f=187 \mathrm{kHz}$. This must be attributed to the WGM's that guide the energy through the line defect. The small value 
of $T_{\text {in }}$ elsewhere at $\mathrm{P}_{2}-\mathrm{P}_{4}$ means that the propagative mode quickly attenuates within the $\Gamma \mathrm{M}$ sample even after only three lines of pillars. This explains the narrowness of the first transmitted spot along the $y$ axis in Fig. 10(d) and its small amplitude.

\section{FURTHER EXPERIMENTAL RESULTS AND ANALYSIS}

(a)

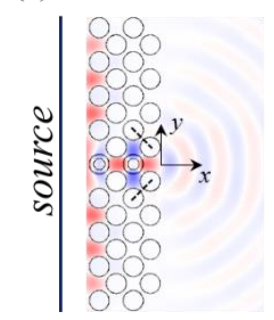

(b) (c)

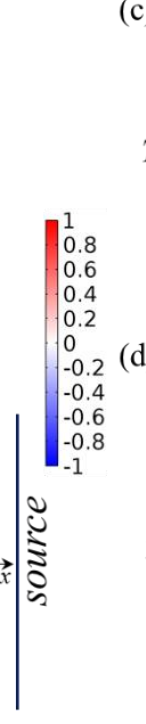

(d)
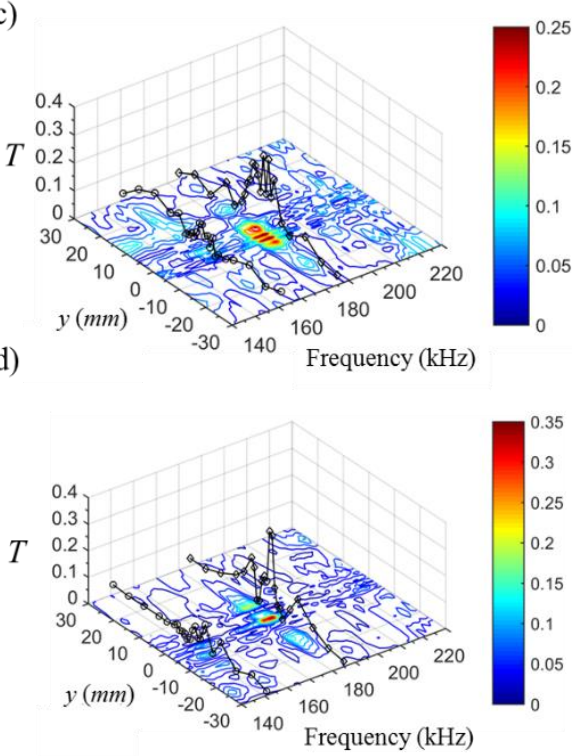

FIG. 11. Calculated distribution of $u_{z}$ at $190 \mathrm{kHz}$ when the elastic source is on the positive (a) and negative (b) part of $x$ axis. (c) and (d) : transmission spectra against the position measured along the $y$ axis at a distance of $6 \mathrm{~mm}$ behind the waveguide relating to the configurations (a) and (b) respectively.

The significant role played in the efficient transmission of Lamb waves both by WGM1 and by the geometry of the solid pillars provides a simple mean to control the beam pattern downstream the PC. Actually, it can inferred that adding lines of pillars to the ГМ sample along the $x$ axis, allows the trefoil shape pattern to be conserved provided that the last two columns are the same as in Fig. 6(c). In contrast, a single fan-shaped beam pattern is obtained if the last column is removed, leading to an outlet interface without any hollow pillar in it. Indeed, the two varieties of outlet interfaces can be crafted in the ГМ structure. This can be verified using the numerical simulations shown in Fig. 11(a) where is represented an antisymmetric Lamb plane wave at $f=$ $190 \mathrm{kHz}$ impinging the device at normal incidence: the occurrence of WGM1 in the hollow pillars is clearly observable therein. Upon excitation, the $\mathrm{A}_{0}$ mode reemitted along the $y$ axis by WMG1 is prevented from attaining the substrate by the solid pillars highlighted by the dashed lines, whereas the $\mathrm{A}_{0}$ mode reemitted along the $x$ axis propagates in the spacing between the solid pillars and generate the fan-shaped pattern in the rear of the device. On the other hand, if the elastic source at $190 \mathrm{kHz}$ is on the right hand side as it is reproduced in Fig. 11(b), the trefoil shape pattern is obtained behind the waveguide owing to the interaction between WGM1 in the hollow pillars and the neighboring solid pillars, similarly to the situation described by Fig. 6(d). Although based on a different mechanism, the trefoil shape pattern and its transferring with the single shape pattern, shows an acoustic counterpart of the angular band gap of light waves in photonic crystals [32]; therefore they suggest potential applications in the spatial 
filtering/modulating of $\mathrm{A}_{0}$ mode as well as other elastic waves in future.

We have fabricated the sample designed in Figs. 11(a) and 11(b) and measured the transmission spectrum of the Lamb waves in the same manner as above. The results recorded $6 \mathrm{~mm}$ behind the PC along a line parallel to the elastic source are displayed in Figs. 11(c) and 11(d) which correspond to the configurations in Figs. 11(a) and 11(b) respectively. We can see one beam behind the waveguide at $186 \mathrm{kHz}$ in Fig. 11(c) and three beams separated by two narrow dips at $x=-3 \mathrm{~mm}$ and $x=5 \mathrm{~mm}$ in Fig. 11(d), both in good agreement with the numerical results. It should be noted that the transmission resulting from the bending vibrations around $f=154 \mathrm{kHz}$ almost disappears from the spectrum $(T<0.1)$, which is also confirmed by the simulations. We attribute that to the internal reflections ensuing from the larger number of lines of scatters in the PC.

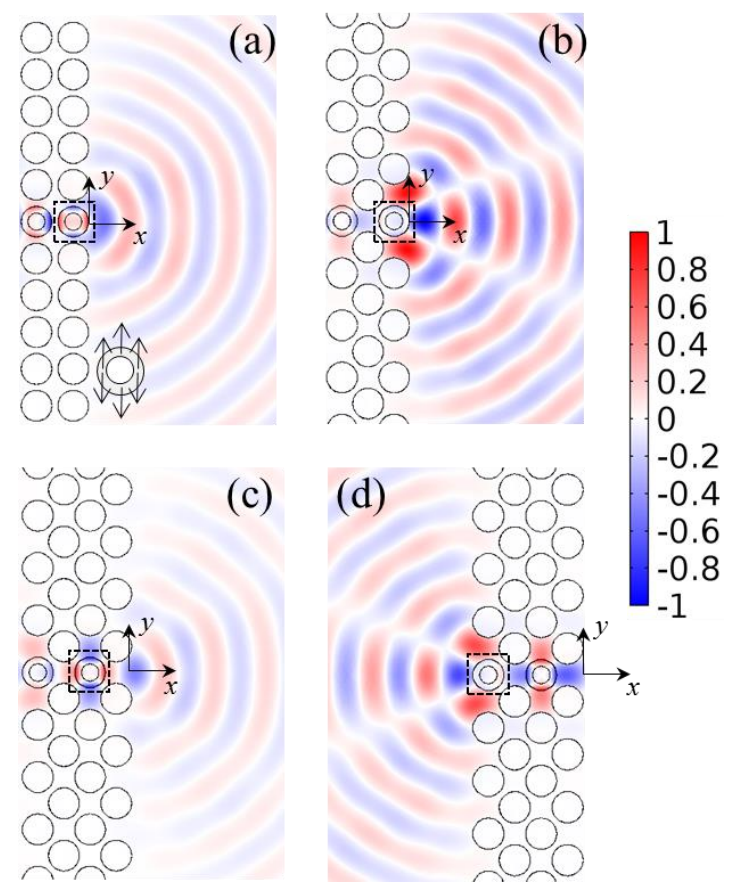

FIG. 12. Out of plane component $u_{z}$ computed when a unit force polarized along the $y$ axis is applied on the top of last hollow pillar in the guide, symmetrically to the $x$ axis (see inset in panel (a)) for (a) $\Gamma X$ sample at $f=192 \mathrm{kHz}$, (b) $\Gamma M$ sample at $f=190 \mathrm{kHz}$, (c) asymmetric $\Gamma M$ sample on the right pillar at $f=190 \mathrm{kHz}$, and (d) asymmetric $\Gamma M$ sample on the left pillar at $f=190 \mathrm{kHz}$.

To further demonstrate that the pattern behind the PC results from the interaction between the quadrupolar reemitted wave and the Lamb wave scattered by the solid pillars at the edges of the guide, let us put into vibration at the frequency of WGM1 the last hollow pillar in the guide. To this end, a harmonic unit force polarized along the $y$ direction is applied on top of the last hollow pillar in the guide (underlined by a square in Fig. 12(a)). Figure 12 shows the calculated distribution of $u_{z}$ for (a) ГX sample, (b) ГМ sample, (c) asymmetric ГМ sample on the right pillar, or (d) asymmetric ГM sample on the left pillar. The generation of WGM1 in the hollow pillars is clearly visible in Fig. 12(a) and the displacement field in the substrate perfectly matches the one induced by the incident plane wave and depicted in Fig. 5(d). The same conclusion holds for the symmetric ГМ sample in Fig. 12(b) which produces the same 
displacement field as the one shown in Fig. 6(d) as well as it is the case for the asymmetric ГМ sample in Figs. 12(c) and 12(d) which gives rise to the same patterns as the ones displayed in Figs. 11(a) and 11(b), respectively.

\section{CONCLUSION}

In summary, we put in evidence both numerically and experimentally the achievements of compact waveguides made of hollow pillars embedded in an array of solid pillars on a plate. We propose with this work a new way to tune the displacement field in the substrate downstream the guide owing to the quadrupolar WGM's. Based on the modal analysis of the WGM's, we have assembled two waveguides with the guiding direction parallel to the $\Gamma \mathrm{X}$ and the $Г \mathrm{M}$ direction, respectively. We then used both the air-coupled and the laser ultrasonic techniques to investigate the transmission spectra of $\mathrm{A}_{0}$ mode. The experimental results in good agreement with the numerical simulations have highlighted two different mechanisms for the transmission of the zero order antisymmetric Lamb wave. At the frequency of the WGM of the hollow pillars, the transmission relates to the reemission of an antisymmetric Lamb wave along the waveguide whereas the bending of the pillar is involved at lower frequency. The measured mapping of $u_{z}$, together with the result of the simulations, allowed us to tune the guided beam pattern behind the $Г \mathrm{X} / Г \mathrm{M}$ sample by means of the quadrupolar WGM but without any contribution of the propagative mode. We then numerically investigated the wave reemitted at the WGM frequency and scattered by a single pillar. In particular, we shown how it affects the beam patterns downstream the $\Gamma \mathrm{X} / \Gamma \mathrm{M}$ sample. We also established the guiding of Lamb waves along the longer waveguides both numerically and experimentally, leading to narrower passing spots with reduced transmissivity in comparison to the short structures. In particular, we built a $Г \mathrm{M}$ sample whose symmetry was broken to get different guided patterns whether the waves are incident from one side of the sample or the other. Our results suggest that WGM could be used in the future in waveguides featuring more sophisticated shapes or even in configurable waveguides. These investigations may be further extended to the higher order of WGM's to achieve more advanced waveguides, or to build acoustic circuits for in-chip devices that can be of great interest in several fields, such as telecommunications, sensors, and medical imaging.

\section{Acknowledgements}

This work is supported by the National Natural Science Foundation of China under Grand No. 11602174, also by the Fundamental Research Funds for the Central Universities under Grand No. 0200219230 and No. 2016KJ008. 


\section{References}

[1] A. Sukhovich, B. Merheb, K. Muralidharan, J. O. Vasseur, Y. Pennec, P. A. Deymier, and J. H. Page, Experimental and theoretical evidence for subwavelength imaging in phononic crystals, Phys. Rev. Lett. 102, 154301 (2009).

[2] X.-F. Li, X. Ni, L. Feng, M.-H. Lu, C. He, and Y.-F. Chen, Tunable unidirectional sound propagation through a sonic-crystal-based acoustic diode, Phys. Rev. Lett. 106, 084301 (2011).

[3] T. Zhang, Y. Cheng, J.-Z. Guo, J.-Y. Xu, and X.-J. Liu, Acoustic logic gates and Boolean operation based on self-collimating acoustic beams, Appl. Phys. Lett. 106, 113503 (2015).

[4] O. A. Kaya, A. Cicek, and B. Ulug, Self-collimated slow sound in sonic crystals, J. Phys. D: Appl. Phys. 45, 365101 (2012).

[5] Y. Guo, D. Brick, M. Großmann, M. Hettich, and T. Dekorsy, Acoustic beam splitting at low GHz frequencies in a defect-free phononic crystal, Appl. Phys. Lett. 110, 031904 (2017).

[6] J. Zhao, B. Bonello, R. Marchal, and O. Boyko, Beam path and focusing of flexural Lamb waves within phononic crystal-based acoustic lenses, New J. Phys. 16, 063031 (2014).

[7] Y. Pennec, B. Djafari-Rouhani, J. O. Vasseur, A. Khelif, and P. A. Deymier, Tunable filtering and demultiplexing in phononic crystals with hollow cylinders, Phys. Rev. E 69, 046608 (2004).

[8] J. O. Vasseur, P. A. Deymier, B. Djafari-Rouhani, Y. Pennec, and A-C. Hladky-Hennion, Absolute forbidden bands and waveguiding in two-dimensional phononic crystal plates, Phys. Rev. B 77, 085415 (2008).

[9] Y. Pennec, B. Djafari-Rouhani, H. Larabi, J. O. Vasseur, and A. C. Hladky-Hennion, Low-frequency gaps in a phononic crystal constituted of cylindrical dots deposited on a thin homogeneous plate, Phys. Rev. B 78, 104105 (2008).

[10] T.-C. Wu, T.-T. Wu, and J.-C. Hsu, Waveguiding and frequency selection of Lamb waves in a plate with a periodic stubbed surface, Phys. Rev. B 79, 104306 (2009).

[11] J.-H. Sun and T.-T. Wu, Propagation of surface acoustic waves through sharply bent twodimensional phononic crystal waveguides using a finite-difference time-domain method, Phys. Rev. B 74, 174305 (2006).

[12] S. Benchabane, O. Gaiffe, R. Salut, G. Ulliac, V. Laude, and K. Kokkonen, Guidance of surface waves in a micron-scale phononic crystal line-defect waveguide, Appl. Phys. Lett. 106, 081903 (2015).

[13] M. Oudich, B. Djafari-Rouhani, Y. Pennec, M. B. Assouar, and B. Bonello, Negative effective mass density of acoustic metamaterial plate decorated with low frequency resonant pillars, J. Appl. Phys. 116, 184504 (2014).

[14] M. Rupin, F. Lemoult, G. Lerosey, and P. Roux, Experimental demonstration of ordered and disordered multiresonant metamaterials for Lamb waves, Phys. Rev. Lett. 112, 234301 (2014).

[15] E. G. Williams, P. Roux, M. Rupin, and W. A. Kuperman, Theory of multiresonant metamaterials for A0 Lamb waves, Phys. Rev. B 91, 104307 (2015).

[16] Y. Jin, N. Fernez, Y. Pennec, B. Bonello, R. P. Moiseyenko, S. Hémon, Y. Pan, and B. DjafariRouhani, Tunable waveguide and cavity in a phononic crystal plate by controlling whispering-gallery modes in hollow pillars, Phys. Rev. B 93, 054109 (2016).

[17] Y.-F. Wang, T.-T. Wang, and Y.-S. Wang, V. Laude, Reconfigurable phononic-crystal circuits formed by coupled acoustoelastic resonators, Phys. Rev. Applied 8, 014006 (2017).

[18] J. Zhao, B. Bonello, and O. Boyko, Focusing of the lowest-order antisymmetric Lamb mode behind a gradient-index acoustic metalens with local resonators, Phys. Rev. B 93, 174306 (2016). 
[19] D. Zhang, J. Zhao, B. Bonello, F. Zhang, W. Yuan, Y. Pan, and Z. Zhong, Investigation of surface acoustic wave propagation in composite pillar based phononic crystals within both local resonance and Bragg scattering mechanism regimes, J. Phys. D: Appl. Phys. 50, 435602 (2017).

[20] F. Lapique and K. Redford, Curing effects on viscosity and mechanical properties of a commercial epoxy resin adhesive, Int. J. Adhe. \& Adhe. 22, 337 (2002).

[21] I. Stewart, A. Chambers, and T. Gordon, The cohesive mechanical properties of a toughened epoxy adhesive as a function of cure level, Int. J. Adhe. \& Adhe. 27, 277 (2007).

[22] E. Coffy, S. Euphrasie, M. Addouche, P. Vairac, and A. Khelif, Evidence of a broadband gap in a phononic crystal strip, Ultrasonics 78, 51 (2017).

[23] B. Sturman and Ingo Breunig, Acoustic whispering gallery modes within the theory of elasticity, J. Appl. Phys. 118, 013102 (2015).

[24] D. Zhang, J. Zhao, B. Bonello, L. Li, J. Wei, Y. Pan, and Z. Zhong, Air-coupled method to investigate the lowest-order antisymmetric Lamb mode in stubbed and air-drilled phononic plates, AIP Adv. 6, 085021 (2016).

[25] R. Marchal, O. Boyko, B. Bonello, J. Zhao, L. Belliard, M. Oudich, Y. Pennec, and B. DjafariRouhani, Dynamics of confined cavity modes in a phononic crystal slab investigated by in situ timeresolved experiments, Phys. Rev. B 86, 224302 (2012).

[26] Y. Jin, B. Bonello, R. P. Moiseyenko, Y. Pennec, O. Boyko, and B. Djafari-Rouhani, Pillar-type acoustic metasurface, Phys. Rev. B 96, 104311 (2017).

[27] M. Ghasemi Baboly, A. Raza, J. Brady, C. M. Reinke, Z. C. Leseman, and I. EI-Kady, Demonstration of acoustic waveguiding and tight bending in phononic crystals, 109, 183504 (2016). [28] Y. Jin, Y. Pennec, Y. Pan, and B. Djafari-Rouhani, Phononic crystal plate with hollow pillars connected by thin bars, J. Phys. D: Appl. Phys. 50, 035301 (2017).

[29] Y.-F. Zhu, X.-Y. Zou, B. Liang, and J.-C. Cheng, Acoustic one-way open tunnel by using metasurface, Appl. Phys. Lett. 107, 113501 (2015).

[30] M. Oudich, S. El-Jallal, Y. Pennec, B. Djafari-Rouhani, J. Gomis-Bresco, D. Navarro-Urrios, C. M. Sotomayor Torres, A. Martínez, and A. Makhoute, Optomechanic interaction in a corrugated phoxonic nanobeam cavity, Phys. Rev. B 89, 245122 (2014).

[31] M. R. Foreman, J. D. Swaim, and F. Vollmer, Whispering gallery mode sensors, Adv. Opt. Photonics 7, 168 (2015).

[32] L. Maigyte and K. Staliunas, Spatial filtering with photonic crystals, Appl. Phys. Rev. 2, 011102 (2015) 